\title{
Exploring for New Physics using Charged Lepton Flavor Violation
}

\author{
Douglas Glenzinski* \\ Fermi National Accelerator Laboratory \\ P.O. Box 500, Batavia, IL 60510, USA \\ E-mail: douglasgefnal.gov
}

\begin{abstract}
The neutrino-less conversion of charged leptons, e.g. $\mu \rightarrow \mathrm{e} \gamma, \mu \rightarrow \mathrm{eee}, \mu \mathrm{N} \rightarrow \mathrm{eN}$, has never been observed. These Charged Lepton Flavor Violating processes offer deep probes of new physics parameter space. Next generation experiments offer sensitivities that are orders of magnitude better than current limits. An overview of planned experiments is provided, concentrating on experiments utilizing intense muon sources, where the largest improvements are expected. These experiments will probe effective new physics mass scales up to $10^{4} \mathrm{TeV} / \mathrm{c}^{2}$, well beyond what can be directly probed at colliders.
\end{abstract}

The International Conference on B-Physics at Frontier Machines - BEAUTY2018

6-11 May, 2018

La Biodola, Elba Island, Italy

\section{${ }^{*}$ Speaker}




\section{Introduction}

Flavor violation, or mixing, has been observed in quarks and neutrinos, so it is natural to expect flavor violating effects among the charged leptons as well. Indeed, there is no known global symmetry that requires lepton flavor conservation. In fact, once neutrino mass is introduced, the $(v-)$ Standard Model provides a mechanism for charged lepton flavor violation (CLFV) as depicted in Figure 1. However, the rate is suppressed by factors proportional to $\left(\Delta \mathrm{m}_{\mathrm{ij}}^{2} / \mathrm{M}^{2}{ }_{\mathrm{W}}\right)^{2}$, where $\Delta \mathrm{m}^{2}{ }_{\mathrm{ij}}$ is the mass-squared difference between the $i^{t h}$ and $j^{\text {th }}$ neutrino mass eigenstates, and is estimated to be extremely small, for example $\operatorname{BR}(\mu \rightarrow \mathrm{e} \gamma)<10^{-54}[1]$. However, many extensions to the Standard Model predict large charged lepton flavor violating (CLFV) effects that could be observed in next generation CLFV experiments [2].

There is global interest in pursuing discovery science via CLFV, with new experiments planned in Europe, Asia, and the Americas. Table 1 compares the expected sensitivity of next generation experiments to current best limits for some CLFV processes. Significant improvements are expected across a wide variety of processes in the next decade. The largest improvements, by factors as large as 10,000, are expected in experiments searching for CLFV transitions using muons.

\section{CLFV searches using muons ${ }^{1}$}

There are three primary muon-to-electron transitions used to search for $\mathrm{CLFV}^{2}$ : a muon decaying into an electron plus photon, $\mu^{+} \rightarrow \mathrm{e}^{+} \gamma$, a muon decaying into three electrons, $\mu^{+} \rightarrow \mathrm{e}^{+} \mathrm{e}^{+} \mathrm{e}^{-}$ and direct muon-to-electron conversion via an interaction with a nucleus, $\mu \mathrm{N} \rightarrow \mathrm{e} \mathrm{N}$. The current best limits for these transitions are $\mathrm{BR}\left(\mu^{+} \rightarrow \mathrm{e}^{+} \gamma\right)<4.2 \times 10^{-13}[5], \mathrm{BR}\left(\mu^{+} \rightarrow \mathrm{e}^{+} \mathrm{e}^{+} \mathrm{e}^{-}\right)<1 \times 10^{-12}[6]$, and $\mathrm{R}_{\mu \mathrm{e}}(\mathrm{Au})<7 \times 10^{-13}[7]$ at $90 \% \mathrm{CL}^{3}$. Next generation experiments are being constructed to have sensitivities significantly beyond these limits. These experiments are described in the next sections.

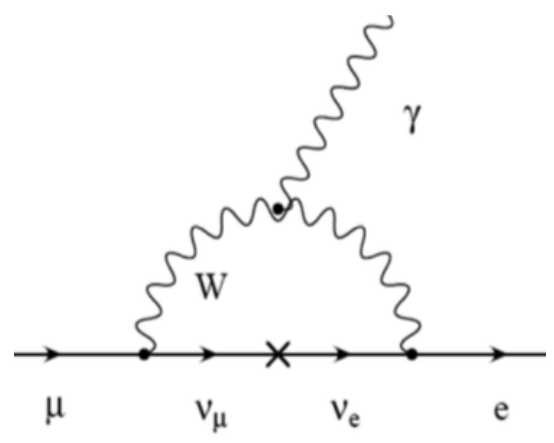

Figure 1: Once neutrino masses are included in the Standard Model, CLFV transitions are allowed via neutrino mixing in loops. An example is provided above for the $\mu \rightarrow$ er transition.

\footnotetext{
${ }^{1}$ A summary of CLFV searches using $\tau$ decays was provided by A. Luciani during the conference.

${ }^{2}$ Muonium oscillations, $\mu^{+} \mathrm{e}^{-} \rightarrow \mu^{-} \mathrm{e}^{+}$where the muon and electron form a bound state, can also be used to set limits on CLFV interactions [4] but are not discussed further in this report.

${ }^{3}$ By convention, $\mathrm{R}_{\mu \mathrm{e}}$ is the $\mu$-to-e conversion rate normalized to the rate of standard muon nuclear capture.
} 


\begin{tabular}{|l|l|c|}
\hline \multicolumn{1}{|c|}{ Process } & Current Limit & Next Generation Exp. \\
\hline$\tau \rightarrow \mu \eta$ & BR $<6.5 \times 10^{-8}$ & \\
\hline$\tau \rightarrow \mu \gamma$ & BR $<4.4 \times 10^{-8}$ & $10^{-9}-10^{-10}$ (Belle II, LHCb) \\
\hline$\tau \rightarrow \mu \mu \mu$ & BR $<2.1 \times 10^{-8}$ & \\
\hline$\tau \rightarrow$ eee & BR $<2.7 \times 10^{-8}$ & NA62 \\
\hline $\mathrm{K}_{\mathrm{L}} \rightarrow \mathrm{e} \mu$ & $\mathrm{BR}<4.7 \times 10^{-12}$ & \\
\hline $\mathrm{K}^{+} \rightarrow \pi^{+} \mathrm{e}^{-} \mu^{+}$ & $\mathrm{BR}<1.3 \times 10^{-11}$ & LHCb, Belle II \\
\hline $\mathrm{B}^{0} \rightarrow \mathrm{e}^{+}$ & $\mathrm{BR}<2.8 \times 10^{-9}$ & \\
\hline $\mathrm{B}^{+} \rightarrow \mathrm{K}^{+} \mathrm{e} \mu$ & $\mathrm{BR}<9.1 \times 10^{-8}$ & $10^{-14}$ (MEG) \\
\hline$\mu^{+} \rightarrow \mathrm{e}^{+} \gamma$ & $\mathrm{BR}<4.2 \times 10^{-13}$ & $10^{-16}$ (PSI) \\
\hline$\mu^{+} \rightarrow \mathrm{e}^{+} \mathrm{e}^{+} \mathrm{e}^{-}$ & $\mathrm{BR}<1.0 \times 10^{-12}$ & $10^{-17}$ (Mu2e, COMET) \\
\hline$\mu^{-} \mathrm{N} \rightarrow \mathrm{e} \mathrm{e}^{-}$ & $\mathrm{R}_{\mu \mathrm{e}}<7.0 \times 10^{-13}$ & \multicolumn{2}{|c|}{} \\
\hline
\end{tabular}

Table 1: For a sampling of CLFV processes, the expected sensitivities of future experiments are compared to current limits. The current limits are taken from [3].

These three $\mu \rightarrow \mathrm{e}$ transitions provide complementary sensitivity to new sources of CLFV. The observed rates will depend on the details of the underlying new physics model, as illustrated in Figure 2. For models in which CLFV rates are dominated by $\gamma$-penguin diagrams the $\mu \rightarrow \mathrm{e} \gamma$, transition rate is expected to be $\sim 10^{2}$ times larger than the $\mu \rightarrow$ eee and $\mu \mathrm{N} \rightarrow \mathrm{eN}$ rates. On the other hand, if the CLFV rates are dominated by $\mathrm{Z} / \mathrm{h}$-penguin diagrams, or if tree level contributions are allowed (e.g. lepto-quark, $Z^{\prime}$ ), then the $\mu \rightarrow$ e $\gamma$ rate is suppressed and $\mu \rightarrow$ eee and $\mu \mathrm{N} \rightarrow \mathrm{eN}$ rates can instead be largest. Thus, by measuring and comparing the rates of all three processes, significant information about the underlying new physics operators can be obtained and used to differentiate among various new physics models. This is discussed further in References [2][8].
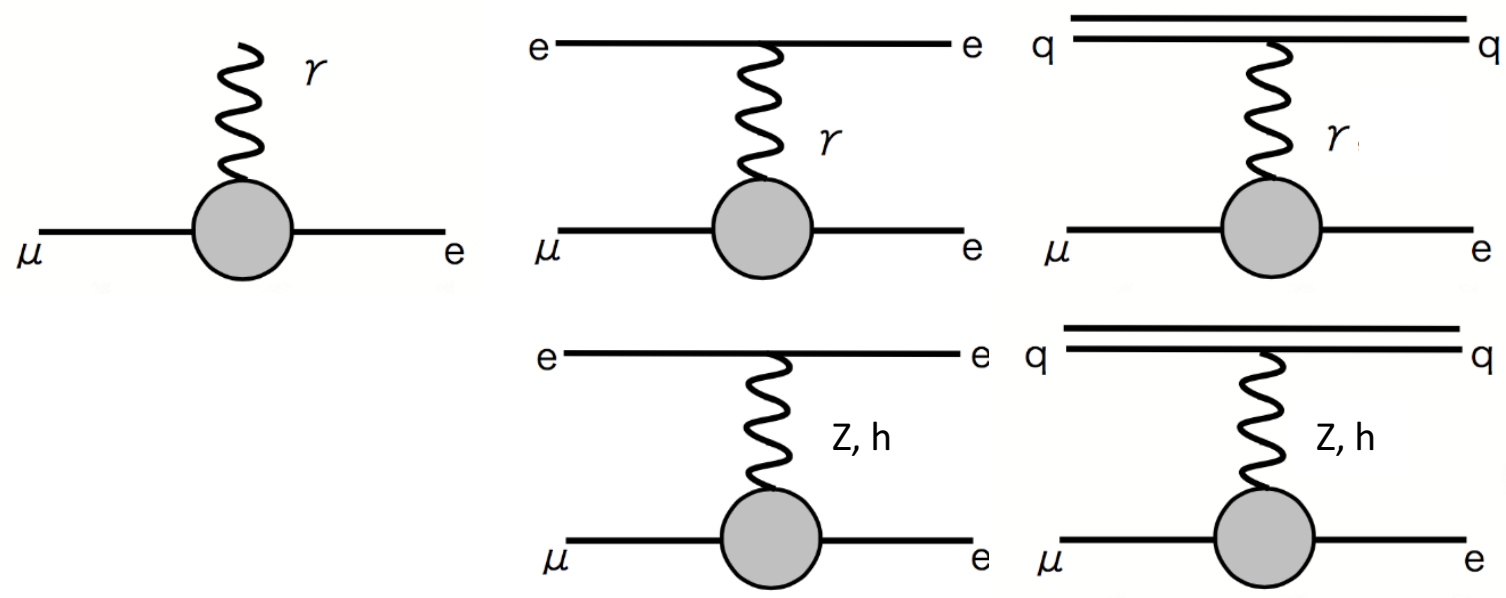

Figure 2: One loop diagrams for $\mu \rightarrow e \gamma$ (left), $\mu \rightarrow e e e$ (mid), and $\mu N \rightarrow e N$ (right) are depicted. The rates of these processes can be compared to differentiate among new physics models. 


\subsection{Experimental methodology}

The same basic experimental methodology is employed for all three CLFV $\mu \rightarrow$ e processes. The experiment beam lines begin by producing low momentum pions by colliding a beam of protons onto a pion production target. The resulting pions are then transported through a decay volume and their decay muons are collected. These experiments require low momentum muons, typically with momenta less than $50 \mathrm{MeV} / \mathrm{c}$, in order to stop them in thin targets at the center of their experimental apparatus. At these low momenta, muons stop in a $\sim 1 \mathrm{~mm}$ or less of material - in stark contrast to the high momentum muons at colliders that penetrate the entire detector volumes. These experiments require high intensity muon beams - current experiments achieve about $10^{7} \mu$-stops/s. Next generation experiments require even higher rates of stopped muons. The experimental apparatus is designed to precisely determine the momentum, energy, and timing of particles originating from the muon stopping target. Because these experiments aim for such extreme sensitivities, the experimental apparatus is customized to the final state of interest.

\subsection{Experiments searching for $\mu^{-} \mathrm{N} \rightarrow \mathrm{e}^{-} \mathrm{N}$}

Experiments searching for direct muon-to-electron conversion require a $\mu^{-}$beam. Once stopped, the $\mu^{-}$forms a muonic atom with a nearby nucleus. Since the mass of the muon is much heavier than the electron, the orbital radius is much smaller resulting in a significant overlap of the muon and nuclear wavefunctions. This large overlap enhances the sensitivity to CLFV processes via interactions with the nucleus. It also modifies the muon lifetime. Once captured in orbit around the nucleus, the muon will do one of three things: decay-in-orbit, $\mu \mathrm{N}(\mathrm{A}, \mathrm{Z}) \rightarrow \mathrm{e}^{-}$ $v v \mathrm{~N}(\mathrm{~A}, \mathrm{Z})$, capture on the nucleus, $\mu \mathrm{N}(\mathrm{A}, \mathrm{Z}) \rightarrow v \mathrm{~N}^{*}(\mathrm{~A}, \mathrm{Z}-1)$, or directly convert into an electron, $\mu^{-} \mathrm{N}(\mathrm{A}, \mathrm{Z}) \rightarrow \mathrm{e}^{-\mathrm{N}}(\mathrm{A}, \mathrm{Z})$. The decay process is a significant source of background for these experiments, the capture process is used for normalization, and the conversion process is the signal. The relative contributions of these processes and the lifetime of the muonic atom depends on the stopping nucleus. Next generation experiments plan to use an aluminum stopping target for which the lifetime of the muonic atom is $864 \mathrm{~ns}, 39 \%$ of the muons will decay in orbit, and $61 \%$ will capture on the nucleus. The capture process produces photons, neutrons, protons, and deuterons and is a significant source of rate in the experiment detectors. The signal conversion process is dominated by coherent interactions with the nucleus to provide a two-body final state yielding a clean experimental signature. The outgoing electron is mono-energetic with an energy near the muon mass less corrections for binding energy and nuclear recoil. For an aluminum stopping target the energy of conversion electrons is $\mathrm{E}_{\mu \mathrm{e}}=104.97 \mathrm{MeV}$, well above the energy of most electrons from decay-in-orbit background events.

Significant sources of background events can arise from decay-in-orbit electrons, from pion interactions in the stopping target, and from cosmic rays that decay in flight or interact in material to produce an electron with energy near $\mathrm{E}_{\mu \mathrm{e}}$. If the energy of the initial proton beam is above the anti-proton production threshold, annihilations of the anti-protons can contribute an additional source of background.

The energy spectrum for decay-in-orbit electrons is shown in Figure 3. The bulk of the spectrum lies below $\mathrm{E}=\mathrm{m}_{\mu} / 2 \sim 52.8 \mathrm{MeV}$, well away from $\mathrm{E}_{\mu \mathrm{e}}$. However, contributions from nuclear recoil give rise to a long tail with an endpoint energy equal to the signal $\mathrm{E}_{\mu \mathrm{e}}$. The background from this process can be kept sufficiently small if the momentum of the electrons can be measured with sufficient precision, $\sigma_{\mathrm{p}}<200 \mathrm{keV} / \mathrm{c}$, and non-Gaussian tails can be kept small. At the energies relevant for these experiments, the momentum resolution is dominated by scattering and straggling effects. For that reason, the tracking detectors must be implemented in very low mass technologies. 


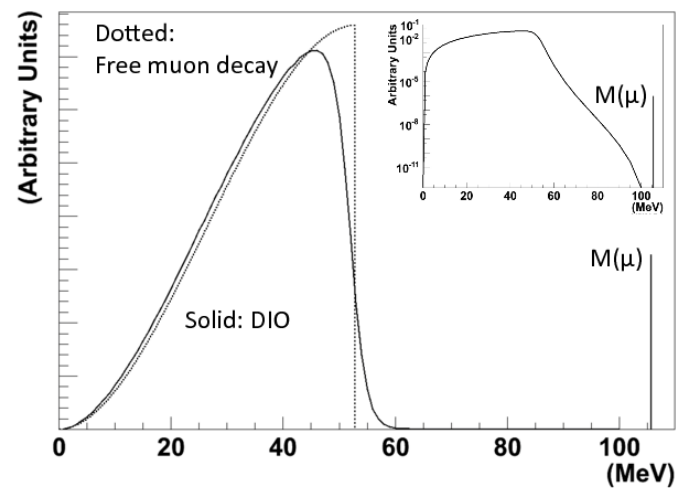

Figure 3: The energy spectrum of electrons from the decay-in-orbit process (DIO). Electrons from the signal conversion process are monoenergetic with an energy $E_{\mu e}$ near the muon mass. The inset shows the DIO spectrum on a logrithmic scale to illustrate the high energy tail that extends out to $E_{\mu e}$.

Pions that survive to the stopping target can interact with the target nuclei to yield a photon with energy as large as $\mathrm{m}_{\pi}$. If the photon converts into an $\mathrm{e}^{+} \mathrm{e}^{-}$pair in material upstream of the tracker, it can yield an electron with energy near $\mathrm{E}_{\mu \mathrm{e}}$. The $\pi \mathrm{N}$ interactions are prompt and experiments take advantage of the relatively long lifetime of the muonic atoms to suppress these backgrounds by utilizing a pulsed proton beam and employing a delayed search window when looking for signal events. The concept is illustrated in Figure 4. This requires a pulsed proton beam, with narrow pulses (full width $<250 \mathrm{~ns}$ ), spaced roughly twice the muonic atom lifetime apart ( $\sim 1700 \mathrm{~ns}$ for an aluminum stopping target), and with very few out-of-pulse protons (the ratio of out-of-pulse to in-pulse protons $<10^{-10}$ ).

Events arising from cosmic ray interactions can be vetoed by identifying incoming cosmic rays with high efficiency ( $>99 \%$ ). This requires a large area, high efficiency, cosmic veto system. Anti-proton induced backgrounds can be mitigated using thin windows in the pion decay volume in order to annihilate the anti-protons far from the stopping target.

The Mu2e experiment at Fermilab [9] and the COMET experiment at JPARC [10] are currently under construction and each aim for a final sensitivity of $\mathrm{R}_{\mu \mathrm{e}}(\mathrm{Al})<7 \times 10^{-17} @ 90 \% \mathrm{CL}$, assuming no signal. Both are based on a concept originated by Lobashev and Djilkabaev [11]. The Mu2e experiment is depicted in Figure 5. Both experiments are designed to be nearly background free with a total mean expected background yield of $<0.5$ events over the full data set. This provides discovery sensitivity for conversion rates larger than a few $10^{-16}$, and would yield striking signals for a wide variety of new physics models, for which rates as large as $10^{-14}$ are predicted.

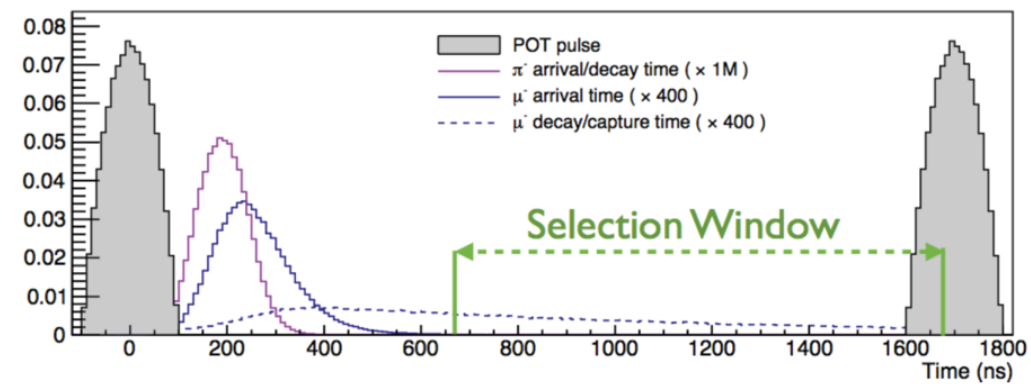

Figure 4: The timing structure for Mu2e. The proton pulses (grey) arrive at the pion production target every $1.7 \mu \mathrm{s}$. The arrival time of muons (pions) at the stopping target is shown in blue (pink). The population of the muonic atoms is also depicted (dash). By employing a selection window delayed by about $700 \mathrm{~ns}$ prompt pion backgrounds are suppressed by about $10^{10}$, while about $50 \%$ of the muonic atoms remain. 


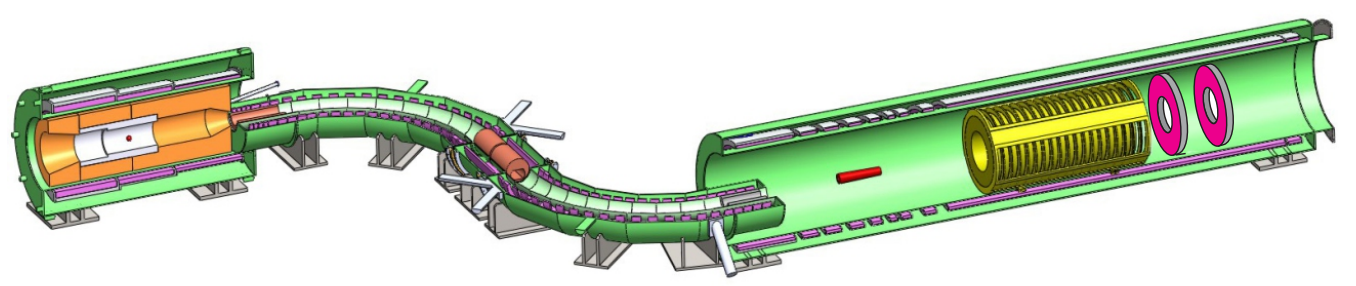

Figure 5: The Mu2e experiment consists of a production solenoid (left) housing the pion production target, an "s"-shaped transport solenoid providing the decay volume, and a detector solenoid (right) including an aluminum muon stopping target, a straw-tube tracker, and a crystal calorimeter. A large area cosmic veto system and beam monitors are not shown. The experiment is about $25 \mathrm{~m}$ end-to-end with a clear bore diameter of about $2 \mathrm{~m}$ in the detector solenoid. A muon stopping rate of about $10^{10} \mu$-stop/s is expected.

\subsection{Experiments searching for $\mu^{+} \rightarrow \mathrm{e}^{+} \gamma$}

Experiments searching for $\mu^{+} \rightarrow \mathrm{e}^{+} \gamma$ decays use a $\mu^{+}$beam to avoid the complications that arise from capture and decay-in-orbit processes in $\mu^{-}$beams. The final state signature is an $\mathrm{e}^{+}$and a $\gamma$, each with $\mathrm{E}=\mathrm{m}_{\mu} / 2$, back-to-back $\left(\theta_{\mathrm{e} \gamma}=180^{\circ}\right)$, and coincident in time. The dominant sources of background arise from radiative muon decay, $\mu^{+} \rightarrow \mathrm{e}^{+} v v \gamma$, and accidental backgrounds from the random combination of a photon from a radiative muon decay or from a positron annihilation in flight with an electron from a separate muon decay $\mu^{+} \rightarrow \mathrm{e}^{+} v \nu$. The accidental background dominates and scales linearly with the timing resolution, and the electron energy resolution, and scales quandratically with the photon energy resolution, the $\theta_{\mathrm{ey}}$ resolution, and the muon stopping rate. Due to the strong dependence of the accidental background on the muon stopping rate, continuous beams are preferred over pulsed beams. The MEG experiment at PSI is depicted in Figure 6 and finished data taking in 2013. It utilized the $\pi \mathrm{E} 5$ beamline, which provided a few $10^{7}$ $\mu$-stop/s, to set the most stringent limit to date, BR $\left(\mu^{+} \rightarrow \mathrm{e}^{+} \gamma\right)<4.2 \times 10^{-13} @ 90 \%$ CL [5]. The experiment is currently being upgraded to further improve the timing, energy, and angular resolutions and to handle $10^{8} \mu$-stop/s. The MEG-II experiment is scheduled to begin commissioning with beam in 2018. A three year physics run is scheduled to begin in 2019. The MEG-II experiment expects to reach a sensitivity of $\mathrm{BR}\left(\mu^{+} \rightarrow \mathrm{e}^{+} \gamma\right)<6 \times 10^{-14} @ 90 \% \mathrm{CL}$ in the absence of signal [12].

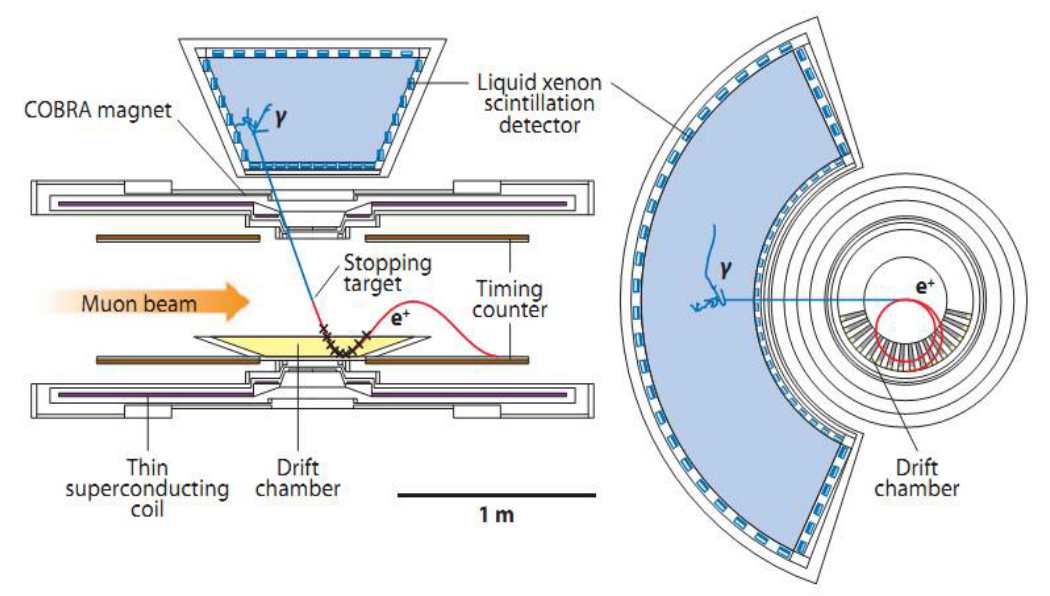

Figure 6 : The MEG experiment consists of a liquid xenon calorimeter and drift chambers for tracking in the central region, and timing counters in the central, backward, and forward regions. The timing and tracking detectors are inside a solonoid magnet with a clear bore radius of about $70 \mathrm{~cm}$. 


\subsection{Experiments searching for $\mu^{+} \rightarrow \mathrm{e}^{+} \mathrm{e}^{+} \mathrm{e}^{-}$}

Experiments searching for $\mu^{+} \rightarrow \mathrm{e}^{+} \mathrm{e}^{+} \mathrm{e}^{-}$also utilize $\mu^{+}$beams. The final state signature is three electrons with energies ranging from $<1 \mathrm{MeV}$ up to a maximum of $\mathrm{E}=\mathrm{m}_{\mu} / 2$, coincident in time, and originating from the same decay vertex. In order to maintain a reasonable acceptance for the three-body final state the experiment must be capable of measuring momenta down to about 10 $\mathrm{MeV} / \mathrm{c}$. The dominant background sources arise from radiative muon decay with the subsequent pair conversion of the photon, $\mu^{+} \rightarrow \mathrm{e}^{+} v v \gamma \rightarrow \mathrm{e}^{+} v v \mathrm{e}^{+} \mathrm{e}^{-}$, and accidental backgrounds from the random combination of electrons from separate decays. The radiative muon decay background can be kept sufficiently small provided the resolution on the electron energy sum, $\Sigma \mathrm{E}_{\mathrm{e}}$, can be kept at about $1.5 \mathrm{MeV}$ or smaller. The accidental background can be suppressed with excellent vertex and timing resolutions.

The $\mu 3 \mathrm{e}$ experiment at PSI [13] will proceed in two phases. Phase-I is currently under construction and aims for a sensitivity of about BR $\left(\mu^{+} \rightarrow \mathrm{e}^{+} \mathrm{e}^{+} \mathrm{e}^{-}\right)<5 \times 10^{-15} @ 90 \% \mathrm{CL}$, assuming no signal. The Phase-I detector is depicted in Figure 7. The experiment shares the same beam line as MEG-II, will utilize $10^{8} \mu$-stop/s, and will require about 300 days of data taking to reach the sensitivity above. The total expected mean background yield is estimated to be $<0.5$ event for the full Phase-I data set. The Phase-I experiment is expected to be ready for commissioning in 2020/1. The $\mu 3 \mathrm{e}$ Phase-II would further extend the detector and would require an upgrade of the muon source to $>10^{9} \mu$-stop/s to improve the sensitivity by another factor of ten or more.

\section{Summary}

Charge Lepton Flavor Violating experiments offer deep probes of new physics parameter space and provide sensitivity in a manner complementary to colliders, neutrino experiments, and dark matter experiments. Significant improvements in sensitivity, by factors ranging from 10 to 10,000 , are expected from several experiments in the next decade. The most sensitive experiments utilize high intensity muon beams. The Mu2e experiment at Fermilab, the COMET experiment at JPARC, and the MEG-II and $\mu 3$ e experiments at PSI are all under construction and expect to begin data taking in the next five years. The current schedules are depicted in Figure 8. These experiments provide complementary sensitivity to a very broad range of new physics models, will probe effective new physics mass scales in the $10^{3}-10^{4} \mathrm{TeV} / \mathrm{c}^{2}$ range, and offer genuine discovery sensitivity. The next decade promises to be exciting as these next-genration CLFV experiments begin taking data.

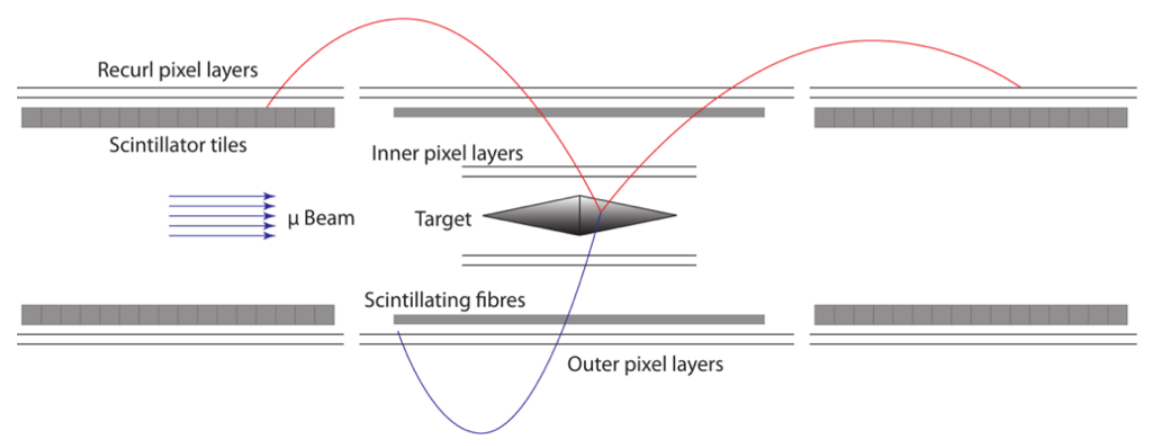

Figure 7: The $\mu 3$ e Phase-I experiment consists of two inner pixel layers immediately outside the stopping target, two additional pixel layers in the central region ("outer pixel layers") and in the forward regions ("recurl pixel layers"), as well as scintillating detectors to provide precision timing information. The timing and tracking detectors are inside a solonoid magnet with a clear bore radius of about $1 \mathrm{~m}$. 


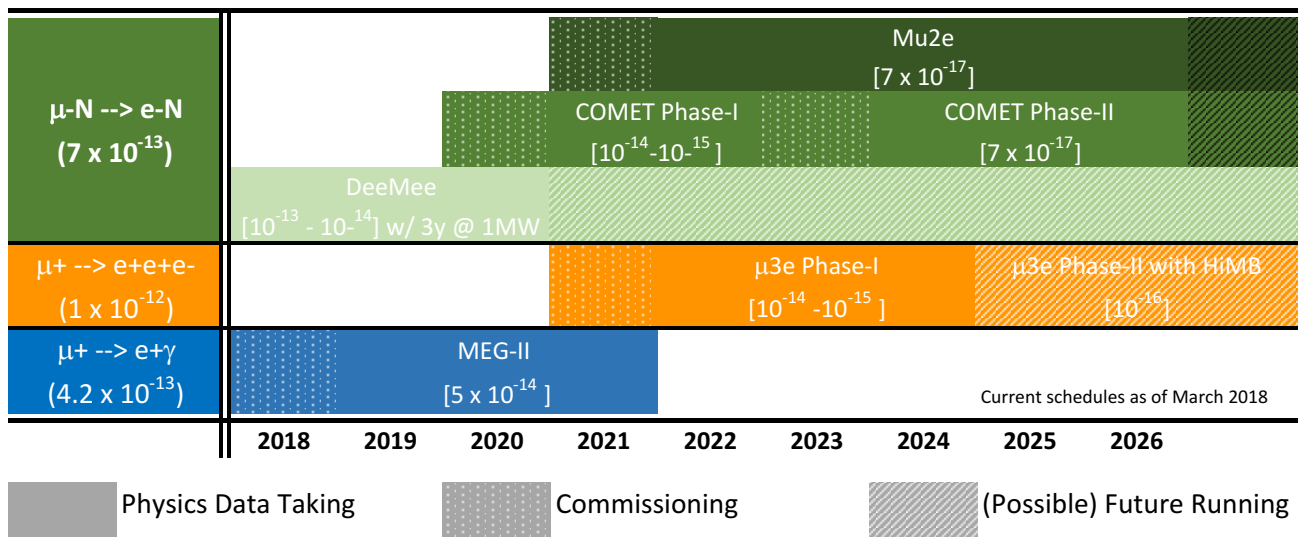

Figure 8 : Expected schedule for the muon CLFV experiments discussed in this report. The current best limits for each process are shown on the left, while the expected sensitivity of the next generation experiments are given on the right. The COMET experiment will occur in two phases as depicted above. The DeeMee experiment utilizes a novel approach to search for muon-to-electron conversion as described in [14]. The $\mu 3$ e Phase-II requires an upgrade to the muon source (HIMS) to reach the target sensitivity.

\section{Acknowledgements}

The author wishes to thank A. Baldini, A. Gaponenko, D. Hitlin, Y. Kuno, J. Miller, T. Mori, and A. Schoening for their help in preparing these conference materials.

\section{References}

[1] S. T. Petcov, Sov. J. Nucl. Phys. 25 (1977) 340.

[2] See, for example L. Calibbi and G. Signorelli, Riv. Nuovo Cimento, 41 (2018) 71.

[3] M. Tanabashi, et al. (Particle Data Group), Phys. Rev. D98 (2018) 030001.

[4] L. Willmann, et al., Phys. Rev. Lett. 82 (1999) 49.

[5] A. M. Baldini, et al. (MEG Collaboration), Eur. Phys. J. C76 (2016) 434.

[6] U. Bellgardt, et al. (SINDRUM Collaboration), Nucl. Phys. B299 (1988) 1.

[7] W. Bertl, et al. (SINDRUM-II Collaboration), Eur. Phys. J. C47 (2006) 337.

[8] V. Cirigliano, et al., Phys. Rev. D80 (2009) 013002.

[9] L. Bartoszek, et al. (Mu2e Experiment), Mu2e Technical Design Report, [arXiv: 1501.05241 ]

[10] R. Abramishvili, et al. (COMET Collaboration), COMET Phase-I Technical Design Report, http://comet.kek.jp/Documents files/PAC-TDR-2016/COMET-TDR-2016 v2.pdf (2016).

[11] R. Djilkibaev and V. M. Lobashev, Sov. J. Nucl. Phys. 49(2) (1989) 384.

[12] A. M. Baldini, et al. (MEG-II Collaboration), Eur. Phys. J. C78 (2018) 380.

[13] A. Blondel, et al. ( $\mu 3$ e Collaboration), Research Proposal for an Experiment to Search for the Decay $\mu \rightarrow e e e$, https://www.psi.ch/mu3e/DocumentsEN/ResearchProposal.pdf (2012).

[14] M. Kinsho, et al. (DeeMee Collaboration), Proposal for an Experimental Search for $\mu$-e Conversion in a Nuclear Field at a Sensitivity of $10^{-14}$ with a Pulsed Proton Beam from RCS, http://deeme.hep.sci.osaka-u.ac.jp/documents/deeme-proposal-r28.pdf/view (2010). 